Revista de Matemática: Teoría y Aplicaciones 2(2): 49-57 (1995)

\title{
SOBRE LOS PUNTOS FIJOS HIPERBÓLICOS DE UN DIFEOMORFISMO LOCAL DEL PLANO
}

\author{
William Alvarado JimÉnEZ ${ }^{1}$
}

\begin{abstract}
Resumen
Se demuestra una generalización del $\lambda$-lema de J. Palis para un caso de intersección no transversal de las variedades estable e inestable de un punto fijo hiperbólico de un difeomorfismo local de clase $\mathcal{C}^{\in}$ del plano en sí mismo.
\end{abstract}

\section{Abstract}

An extension of J. Palis' $\lambda$-lemma is obtained for one case of non-tansversal intersection of the stable and unstable manifolds of an hyperbolic fixed point of a $\mathcal{C}^{\epsilon}$-local diffeomorphism of $\mathbb{R}^{2}$.

\section{Introducción}

Consideremos un difeomeorfismo local $H$ de clase $\mathcal{C}^{\nabla}$ de un vecindario del origen de coordenadas $0 \in \mathbb{R}^{n}$, que admite a 0 en calidad de punto fijo hiperbólico. En la descomposición invariante $\mathbb{R}^{n}=E^{s} \oplus E^{u}$ inducida por el isomorfismo lineal $D H(0)$, consideramos dos bolas abiertas $B^{s} \subseteq E^{s}$, y $B^{u} \subseteq E^{u}$, contenidas respectivamente en la variedad estable local $W_{l o c}^{s}$, y el la variedad inestable local $W_{l o c}^{u}$ del difeomorfismo $H$ en el punto $0 \in \mathbb{R}^{n}$. Sean $V=B^{s} \times B^{u}, q=(p, 0) \in W_{l o c}^{s} \backslash\{0\}$, y $D^{k}$ un disco de dimensión $k$ igual a la dimensión del subespacio $E^{u}$, que interseca transversalmente la variedad estable local $W_{l o c}^{s}$ en el punto $q$.

El siguiente resultado, debido a J. Palis, se conoce dentro de la teoría de sistemas dinámicos con el nombre de $\lambda$-lema:

\section{Lema ( $\lambda$-lema).}

Sean $V$, q y $D^{k}$ como arriba. Si $D_{n}^{k}$ denota la componente conexa de la intersección $H^{n}\left(D^{k}\right) \cap V$ que contiene al n-ésimo iterado $H^{n}(q)$ del punto $q$ bajo la acción del difeomorfismo $H$, entonces dado $\varepsilon>0$, se puede hallar un número natural $N$, tal que si $n \geq N$, entonces $D_{n}^{k}$ es $\varepsilon \mathcal{C}^{\prime}$-próximo de la bola $B^{u}$.

Este lema de caracter local se utiliza a menudo en el estudio de sistemas dinámicos en dimensiones mayores o iguales a 2. En particular, el $\lambda$-lema se suele utilizar para dar

\footnotetext{
${ }^{1}$ Escuela de Matemática, Universidad de Costa Rica
} 
una demostración "geométrica" del Teorema de Grobman-Hartman, o bien para estudiar el comportamiento de trayectorias en un vecindario de un contorno homoclínico estructuralmente estable ([PALIS-DE MELO $]$ ).

Aunque la formulación del $\lambda$-lema es bastante "técnica", geométricamente este resultado afirma que en un vecindario de un punto fijo hiperbólico de un difeomorfismo local de clase $\mathcal{C}^{\nabla}$, de $0 \in \mathbb{R}^{n}$, si un disco de dimensión igual a la dimensión de la variedad inestable local $W_{l o c}^{u}(0)$ interseca transversalmente a la variedad estable local $W_{l o c}^{s}(0)$, entonces los iterados sucesivos del difeomorfismo "enderezan" el disco, de tal forma que para $n$ suficientemente grande, el $n$-ésimo iterado del disco no sólo estará muy próximo a $W_{l o c}^{u}$, sino que también el espacio tangente del n-ésimo iterado del disco es "casi" paralelo al espacio tangente de la variedad inestable local. En el caso particular del plano, la figura 1 da una idea del comportamiento de los iterados del difeomorfismo $H$.

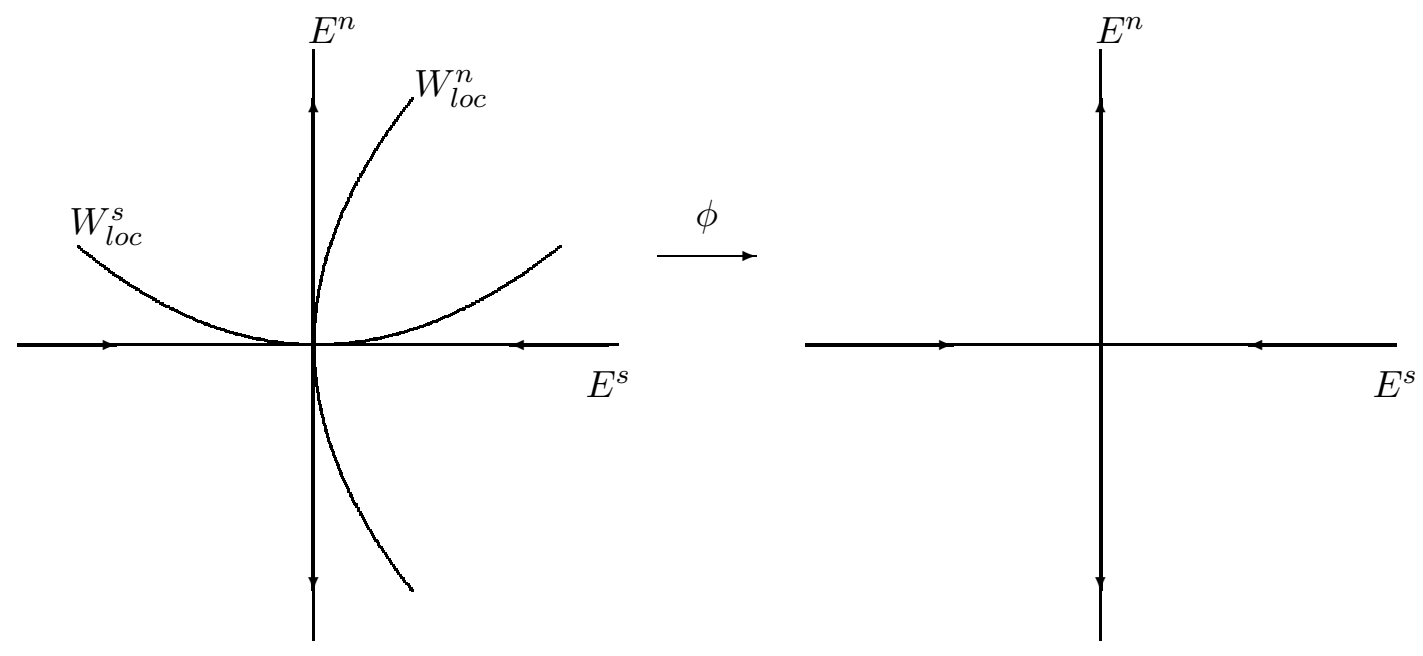

Figura 1:

En el presente trabajo, vamos demostrar, en el plano, un resultado similar al $\lambda$-lema para un punto fijo hiperbólico de un difeomorfismo local de clase $\mathcal{C}^{\epsilon}$, en el caso en que la intersección de una curva $\gamma$ con la variedad estable local $W_{l o c}^{s}$, sin ser necesariamente transversal, satisfaga condiciones de "normalidad" que se definen más adelante.

\section{Resultados preparatorios}

En esta sección vamos a enumerar varios resultados preliminares, que son necesarios para la prueba del teorema principal. Las demostraciones de estas afirmaciones, aunque elementales, son bastante largas, y es por esto que hemos preferido omitirlas, refiriendo al lector interesado a los trabajos [Alvarado], [Ivanov], [Palis-de Melo].

En $\mathbb{R}^{2}$ consideremos un vecindario abierto $O$ del origen de coordenadas, y un difeomorfismo $H: O \longrightarrow \mathbb{R}^{2}$ de clase $\mathcal{C}^{\epsilon}$, que tiene un punto fijo hiperbólico en el origen de coordenadas $(0,0)$.

Vamos a suponer que los valores propios $\alpha$ y $\beta$ del isomorfismo lineal $D H(0)$ verifican 
$|\alpha|<1<|\beta|^{2}$. Además, vamos a suponer, sin pérdida de generalidad, que el producto $\alpha \beta$ es estrictamente menor a 1.

Consideremos la descomposición del plano: $\mathbb{R}^{2}=E^{s} \oplus E^{u}$ inducida por el isomorfismo lineal $D H(0)$. Como se sabe, la variedad estable local del punto $0, W_{l o c}^{s}$ corresponde al gráfico de una aplicación de clase $\mathcal{C}^{\in}$,

$$
\varphi_{s}: B^{s}(0, r) \longrightarrow E^{u}
$$

tal que $\varphi_{s}(0)=0$ y $D \varphi_{s}(0)=0$, donde $B^{s}(0, r) \subseteq E^{s}$ denota la bola abierta de centro 0 y radio $r$.

Análogamente, la variedad inestable local de $0, W_{l o c}^{u}(0)$ se puede ver como el gráfico de una aplicación $\mathcal{C}^{\in}$,

$$
\varphi_{u}: B^{u}(0, r) \longrightarrow E^{s}
$$

que verifica $\varphi_{u}(0)=0$ y $D \varphi_{u}(0)=0$.

Consideremos la aplicación $\varphi$, definida por

$$
\begin{gathered}
\varphi: B^{s}(0, r) \times B^{u}(0, r) \longrightarrow E^{s} \oplus E^{u} \\
\left(x_{s}, x_{u}\right) \mapsto\left(x_{s}-\varphi_{u}\left(x_{u}\right), x_{u}-\varphi_{s}\left(x_{s}\right)\right) .
\end{gathered}
$$

La aplicación $\varphi$ es de clase $\mathcal{C}^{\in}$, y además $D \varphi(0)=I$, donde $I$ denota la aplicación identidad de $\mathbb{R}^{2}$. De esta manera $\varphi$ es un difeomorfismo local de un vecindario de $0 \in \mathbb{R}^{2}$ en $\mathbb{R}^{2}$. Consideremos la composición $\widetilde{H}=\varphi \circ H \circ \varphi^{-1}$. $\widetilde{H}$ es un difeomorfismo local de un vecindario del origen de coordenadas, que satisface $\widetilde{H}(0)=0$ y $D \widetilde{H}(0)=D H(0)$.

De esta manera, hemos probado que la variedad estable local del punto 0 coincide con un vecindario del origen de $E^{s}$, y que la variedad inestable local, con un vecindario del origen de $E^{u}$.

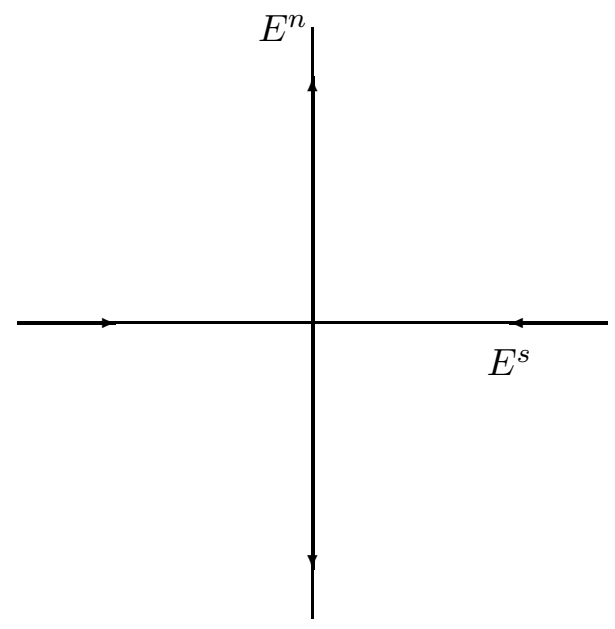

Figura 2:

\footnotetext{
${ }^{2}$ En otras palabras 0 es un punto fijo hiperbólico del tipo silla de montar (saddle-point)
} 
Gracias a esto, podemos asumir que las variedades estable local e inestable local de un punto fijo hiperbólico de un difeomorfismo local $H$, son un vecindario del punto fijo en el subespacio estable y en el subespacio inestable respectivamente de la parte lineal de $H$ en este punto.

Consideremos de nuevo nuestro difeomorfismo local $H$, el cual podemos suponer sin pérdida de generalidad, tiene la forma

$$
H(x, y)=(F(x, y), G(x, y))=(\alpha x+f(x, y), \beta y+g(x, y)),
$$

donde $f, g \in \mathcal{C}^{\in}$ en $O$, satisfacen las condiciones

$$
f(0,0)=g(0,0)=f(0, y)=g(x, 0)=\frac{\partial f}{\partial x}(0,0)=\frac{\partial g}{\partial y}(0,0)=0 .
$$

Las siguientes proposiciones resumen el comportamiento de los iterados de los puntos pertenecientes a un vecindario suficientemente pequeño del origen, bajo la acción del difeomorfismo local $H$.

Primeramente introducimos dos funciones auxiliares $\phi$ y $\psi$ :

$$
\phi(x, y)= \begin{cases}\frac{f(x, y)}{\alpha x} & , \text { si } x \neq 0 \\ 0 & , \text { si } x=0\end{cases}
$$

y

$$
\psi(x, y)= \begin{cases}\frac{g(x, y)}{\beta x} & , \text { si } y \neq 0 \\ 0 & , \text { si } y=0 .\end{cases}
$$

En lo que sigue denotamos por $B(\delta)$ la bola abierta de centro 0 y radio $\delta$.

\section{Proposición 1.}

Existen constantes $\delta_{1}>0$ y $M>0$, tal que si $(x, y) \in B\left(\delta_{1}\right)$, entonces

$$
\begin{aligned}
& |\phi(x, y)|<M(|x|+|y|) \\
& |\psi(x, y)|<M(|x|+|y|) .
\end{aligned}
$$

Sea $\left(x_{0}, y_{0}\right)$ un punto que pertenece a $B\left(\delta_{1}\right)$, y supongamos que los puntos $\left(x_{i}, y_{i}\right)$ definidos por la fórmula

$$
\left(x_{i}, y_{i}\right)=H^{i}\left(x_{0}, y_{0}\right),
$$

donde $H^{i}$ denota el $i$-ésimo iterado de la aplicación $H$, también pertenecen a $B\left(\delta_{1}\right)$, para $i=1, \ldots, n$.

Para cada $i, i=0, \ldots, n-1$, escribimos

$$
\phi_{i}=\phi\left(x_{i}, y_{i}\right), \quad \psi_{i}=\psi\left(x_{i}, y_{i}\right) .
$$


De esta forma, se tiene que, para $k=1, \ldots, n$ :

$$
\begin{aligned}
& x_{k}=\alpha^{k} x_{0} \prod_{i=0}^{k-1}\left(1+\phi_{i}\right) \\
& y_{k}=\beta^{k} y_{0} \prod_{i=0}^{k-1}\left(1+\psi_{i}\right) .
\end{aligned}
$$

\section{Proposición 2.}

Existen $\delta_{2}>0, \delta_{2} \leq \delta_{1}, y$ constantes positivas $\alpha_{1}, \alpha_{2}, \beta_{1}$, independientes de $n$, que verifican

$$
0<\alpha_{1}<|\alpha|<\alpha_{2}<1<\beta_{1}<|\beta|,
$$

tales que si los puntos $\left(x_{i}, y_{i}\right)$ pertenecen a $B\left(\delta_{2}\right)$, para $i=1, \ldots, n$, entonces

$$
\left|x_{0}\right| \alpha_{1}^{n}<x_{n}<\left|x_{0}\right| \alpha_{2}^{n},
$$

$y$

$$
\left|y_{0}\right|<\left|y_{n}\right| \beta_{1}^{-n}
$$

\section{Proposición 3.}

Existen $\delta_{3}>0, \delta_{3} \leq \delta_{2}$, y una constante $M_{1}>0$ independientes de $n$, tales que si los puntos $\left(x_{i}, y_{i}\right)$ pertenecen a $B\left(\delta_{3}\right)$, para $i=1, \ldots, n$, entonces se satisfacen las desigualdades

$$
\begin{aligned}
|\alpha|^{n}\left(1-M_{1} \delta\right)\left|x_{0}\right| & <\left|x_{n}\right|<|\alpha|^{n}\left(1+M_{1} \delta\right)\left|x_{0}\right| \\
|\beta|^{-n}\left(1-M_{1} \delta\right)\left|y_{n}\right| & <\left|y_{0}\right|<|\beta|^{-n}\left(1+M_{1} \delta\right)\left|y_{n}\right|,
\end{aligned}
$$

para cualquier $0<\delta \leq \delta_{3}$.

\section{Proposición 4.}

Sea $M_{1}$ como en la proposición anterior, y sea $0<\delta \leq \delta_{3}$, supongamos que todos los puntos $\left(x_{0}, y_{0}\right), \ldots,\left(x_{n}, y_{n}\right)$ pertenecen a $B(\delta)$, entonces

$$
\begin{aligned}
& 1-M_{1} \delta<\left|\prod_{i=0}^{n-1}\left(1 \pm\left|\phi_{i}\right|\right)\right|<1+M_{1} \delta, \\
& 1-M_{1} \delta<\left|\prod_{i=0}^{n-1}\left(1 \pm\left|\psi_{i}\right|\right)\right|<1+M_{1} \delta,
\end{aligned}
$$

y es posible encontrar $\vartheta=\vartheta\left(n, x_{0}, y_{0}, \delta\right)$ y $\varrho=\varrho\left(n, x_{0}, y_{0}, \delta\right)$ que verifican $\vartheta, \varrho<M_{1} \delta, y$

$$
x_{n}=\alpha^{n} x_{0}(1+\vartheta), \quad y_{0}=\beta^{-n} y_{n}(1+\varrho) .
$$




\section{Proposición 5.}

Existen constantes $0<\delta_{4} \leq \delta_{3}$ y $M_{2}>0$ independientes de $n$ tales que si $0<\delta \leq \delta_{4}$, y si $\left(x_{0}, y_{0}\right), \ldots,\left(x_{n}, y_{n}\right) \in B(\delta)$, entonces

$$
\begin{array}{ll}
\left|\frac{\partial x_{n}}{\partial x_{0}}-\alpha^{n}\right|<|\alpha|^{n} M_{2} \delta, & \left|\frac{\partial x_{n}}{\partial y_{0}}\right|<(\alpha \beta)^{n} M_{2} \delta, \\
\left|\frac{\partial y_{n}}{\partial x_{0}}\right|<M_{2} \delta \quad, & \left|\frac{\partial y_{n}}{\partial y_{0}}-\beta^{n}\right|<|\beta|^{n} M_{2} \delta .
\end{array}
$$

\section{Resultado principal}

En esta sección vamos a considerar de nuevo el difeomorfismo local $H$ introducido en la sección anterior, y vamos a suponer que $H$ está definido en un vecindario $O^{\prime}$ del origen, contenido en la bola $B\left(\delta_{4}\right)$.

Sea $E^{s} \oplus E^{u}$ la descomposición de $\mathbb{R}^{2}$ inducida por el isomorfismo $D H(0): \mathbb{R}^{2} \longrightarrow \mathbb{R}^{2}$. Como vimos más arriba, podemos suponer sin pérdida de generalidad que $O^{\prime}=B^{s} \times B^{u}$, donde $B^{s} \subseteq E^{s}$ y $B^{u} \subseteq E^{u}$ son vecindarios del origen, contenidos en la variedad estable local $W_{l o c}^{s}(0)$ y la variedad inestable local $W_{l o c}^{u}(0)$ del punto $0 \in \mathbb{R}^{2}$. En $E^{s}$ y $E^{u}$ definimos una orientación compatible con la orientación usual de $\mathbb{R}^{2}$, y ponemos

$$
B_{+}^{u}=\left\{x_{u} \in B^{u}: x_{u}>0\right\}, O_{+}^{\prime}=B_{s} \times B_{+}^{u} .
$$

Como se vió más arriba, en $O^{\prime}$ la aplicación $H$ tiene la forma:

$$
H\left(x_{s}, x_{u}\right)=\left(F\left(x_{s}, x_{u}\right), G\left(x_{s}, x_{u}\right)\right)=\left(\alpha x_{s}+f\left(x_{s}, x_{u}\right), \beta x_{u}+g\left(x_{s}, x_{u}\right)\right),
$$

donde $f, g$ son funciones de clase $\mathcal{C}^{\in}$ en $O^{\prime}$, que satisfacen las siguientes condiciones:

$$
f(0,0)=g(0,0)=f(0, y)=g(x, 0)=\frac{\partial f}{\partial x}(0,0)=\frac{\partial g}{\partial y}(0,0)=0 .
$$

Por hipótesis, $O^{\prime} \subseteq B\left(\delta_{4}\right)$, y por lo tanto todas las propiedades enunciadas en las proposiciones $1-5$ se verifican en $O^{\prime}$.

Consideremos el punto $(p, 0) \in B^{s} \backslash\{0\}$, y asumamos que $\gamma=\left\{\left(\gamma_{s}(t), \gamma_{u}(t)\right): t \in\right.$ $[-1,1]\}$ es una curva de clase $\mathcal{C}^{\in}$, tangente a $W_{l o c}^{s}(0)$ en el punto $(p, 0)=\left(\gamma_{s}(0), \gamma_{u}(0)\right)$. Denotemos por $\gamma^{n}$ la componente conexa de $H^{n} \gamma \cap O^{\prime}$ que contiene al punto $H^{n}(p, 0)$.

\section{Definición 6.}

Sea $(0, q) \in B^{u} \backslash\{0\}$. Decimos que una curva $\gamma$ satisface la condición de tangencia normal (abreviada TN) respecto al difeomorfismo $H$ y el punto $(0, q)$, si:

(a) $\gamma_{u}^{\prime}$ tiene un cero aislado en $t=0$, 
(b) para cualquier vecindario $A$ del punto $(p, 0)$, existe un $t_{A} \in[-1,1]$ tal que $\gamma\left(t_{A}\right) \in A$, y $\gamma_{u}\left(t_{A}\right) \neq 0$, si $\beta<0$; o bien $\gamma_{u}\left(t_{A}\right) q>0$, si $\beta>0$.

Observemos que la condición (b) de la definición anterior supone que en caso orientable $(\beta>0)$ cualquier vecindario de $0 \in[-1,1]$, existe un punto $t_{A}$ tal que $\left(\gamma_{s}\left(t_{A}\right), \gamma_{u}\left(t_{A}\right)\right)$ esté en el mismo semiplano que $(0, q)$.

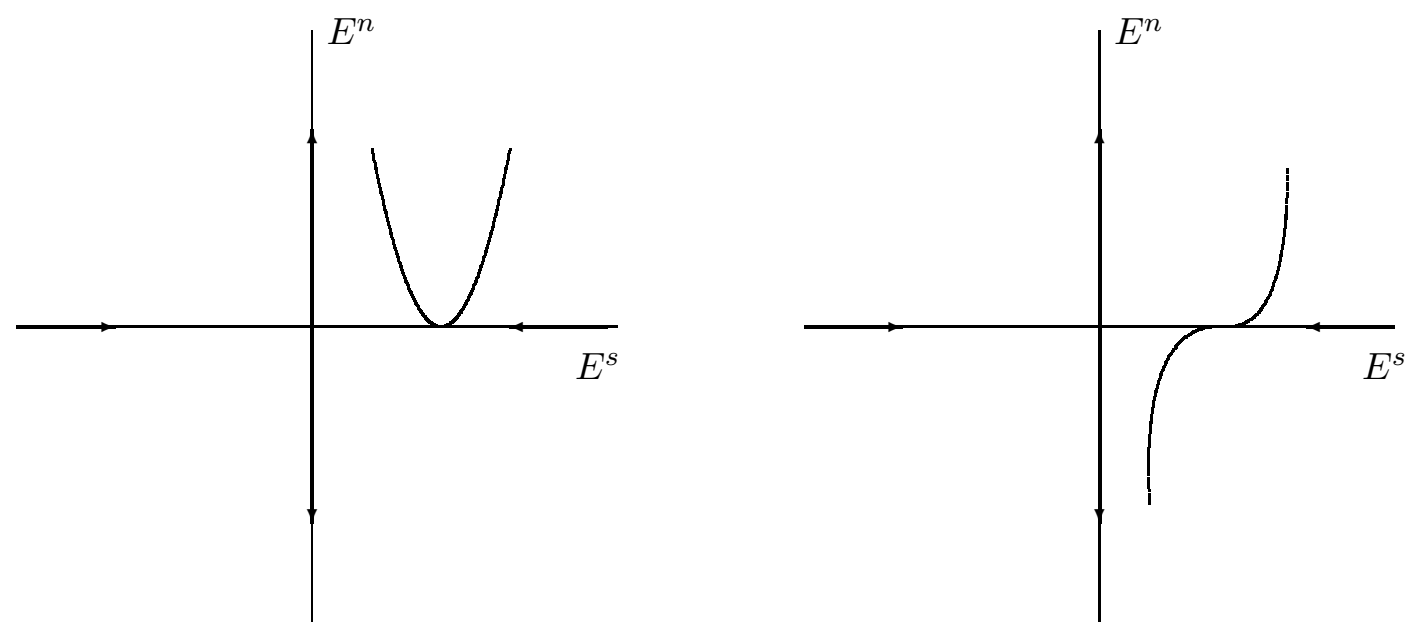

Figura 3:

\section{Teorema 7 .}

Sea $(0, q) \in B_{+}^{u}$. Si la curva $\gamma$ satisface la condición $T N$ respecto al difeomorfismo $H$ y al punto $(0, q)$, entonces para cualquier $q>\varepsilon>0$ existe un $N \in \mathbb{N}$, tal que si $n \geq N$ y el punto $\left(\gamma_{s}(\tilde{t}), \gamma_{u}(\tilde{t})\right)$ pertenece al conjunto $H^{-n}([-\varepsilon, \varepsilon] \times[q-\varepsilon, q+\varepsilon]) \cap \gamma$, entonces

$$
\frac{\left|\tilde{v}_{s}^{n}\right|}{\left|\tilde{v}_{u}^{n}\right|} \leq \varepsilon
$$

donde $\tilde{v}^{n}=\left(\tilde{v}_{s}^{n}, \tilde{v}_{u}^{n}\right)$ denota el vector tangente a la curva $\gamma^{n}$ en el punto $\left(\gamma_{s}^{n}(\tilde{t}), \gamma_{u}^{n}(\tilde{t})\right)$.

(Vale la pena observar que la condición $\left|\tilde{v}_{s}^{n}\right| /\left|\tilde{v}_{u}^{n}\right| \leq \varepsilon$ significa que la inclinación del vector $\tilde{v}^{n}$ respecto al subespacio $E^{u}$ es menor que $\varepsilon$, i.e. que el vector $\tilde{v}^{n}$ es "casi" paralelo a $E^{u}$. Por otra parte, es claro que si el punto $\left(\gamma_{s}^{n}(\tilde{t}), \gamma_{u}^{n}(\tilde{t})\right)$ pertenece a $B\left(\delta_{4}\right)$, entonces la distancia entre dicho punto y la variedad $W_{l o c}^{u}(0)$ se puede hacer tan pequeña como se quiera, si tan solo $n$ es suficientemente grande.)

\section{Demostración.}

Para comenzar observemos que es suficiente considerar el caso $\beta>0$, ya que el caso $\beta<0$ se reduce a éste, si se considera el difeomorfismo $H^{2}$ en lugar de $H$ y la curva $H \gamma$ en vez de $\gamma$. 
Sea $\left(\gamma_{s}^{n}(\tilde{t}), \gamma_{u}^{n}(\tilde{t})\right) \in H^{-n}([-\varepsilon, \varepsilon] \times[q-\varepsilon, q+\varepsilon]) \cap \gamma$. Dado que la curva $\gamma$ satisface la condición TN respecto al difeomorfismo $H$ y el punto $(0, q)$, podemos suponer que $\gamma^{\prime}(\tilde{t}) \neq 0$.

Por definición

$$
\begin{aligned}
\tilde{v}_{s}^{n} & =\frac{d}{d t} \gamma_{s}^{n}(\tilde{t})=\frac{d}{d t} F^{n}\left(\gamma_{s}(\tilde{t}), \gamma_{u}(\tilde{t})\right)= \\
& =\frac{\partial F^{n}\left(\gamma_{s}(\tilde{t}), \gamma_{u}(\tilde{t})\right)}{\partial \gamma_{s}} \cdot \gamma_{s}^{\prime}(\tilde{t})+\frac{\partial F^{n}\left(\gamma_{s}(\tilde{t}), \gamma_{u}(\tilde{t})\right)}{\partial \gamma_{u}} \cdot \gamma_{u}^{\prime}(\tilde{t}), \\
\tilde{v}_{u}^{n} & =\frac{d}{d t} \gamma_{u}^{n}(\tilde{t})=\frac{d}{d t} G^{n}\left(\gamma_{s}(\tilde{t}), \gamma_{u}(\tilde{t})\right)= \\
& =\frac{\partial G^{n}\left(\gamma_{s}(\tilde{t}), \gamma_{u}(\tilde{t})\right)}{\partial \gamma_{s}} \cdot \gamma_{s}^{\prime}(\tilde{t})+\frac{\partial G^{n}\left(\gamma_{s}(\tilde{t}), \gamma_{u}(\tilde{t})\right)}{\partial \gamma_{u}} \cdot \gamma_{u}^{\prime}(\tilde{t}) .
\end{aligned}
$$

Ahora, por la proposición 5, obtenemos:

$$
\begin{aligned}
& \left|\tilde{v}_{s}^{n}\right| \leq \alpha^{n}\left(1+M_{2} \delta_{4}\right)\left|\gamma_{s}^{\prime}(\tilde{t})\right|+(\alpha \beta)^{n} M_{2} \delta_{4}\left|\gamma_{u}^{\prime}(\tilde{t})\right| \\
& \left|\tilde{v}_{u}^{n}\right| \geq\left|\beta^{n}\left(1-M_{2} \delta_{4}\right)\right| \gamma_{u}^{\prime}(\tilde{t})\left|-M_{2} \delta_{4}\right| \gamma_{s}^{\prime}(\tilde{t})|| .
\end{aligned}
$$

Definimos

$$
s_{n}=\sup \left\{\frac{\left|\gamma_{s}^{\prime}(t)\right|}{\left|\gamma_{u}^{\prime}(t)\right|}:\left(\gamma_{s}(t), \gamma_{u}(t)\right) \in H^{-n}([-\varepsilon, \varepsilon] \times[q-\varepsilon, q+\varepsilon])\right\} .
$$

(Observemos que como $\left\{t \in[-1,1]:\left(\gamma_{s}(t), \gamma_{u}(t)\right) \in H^{-n}([-\varepsilon, \varepsilon] \times[q-\varepsilon, q+\varepsilon])\right\}$ es cerrado en $[-1,1]$, entonces $s_{n}<+\infty$.)

Hay tres casos a considerar:

(1) $\beta^{-n} s_{n}$ es acotado cuando $n \rightarrow \infty$.

(2) $\alpha^{n} \beta^{-n} s_{n} \rightarrow \infty$, cuando $n \rightarrow \infty$.

(3) $\alpha^{n} \beta^{-n} s_{n}$ es acotado y $\beta^{-n} s_{n} \rightarrow \infty$, cuando $n \rightarrow \infty$.

(1): Se tiene que:

$$
\begin{aligned}
\frac{\left|\tilde{v}_{s}^{n}\right|}{\left|\tilde{v}_{u}^{n}\right|} & \leq \frac{\alpha^{n}\left(1+M_{2} \delta_{4}\right) s_{n}+(\alpha \beta)^{n} M_{2} \delta_{4}}{\left|\beta^{n}\left(1-M_{2} \delta_{4}\right)-M_{2} \delta_{4} s_{n}\right|}= \\
& =\frac{\alpha^{n} \beta^{-n}\left(1+M_{2} \delta_{4} s_{n}+\alpha^{n} M_{2} \delta_{4}\right.}{\left|\left(1-M_{2} \delta_{4}\right)-\beta^{-n} M_{2} \delta_{4} s_{n}\right|},
\end{aligned}
$$

y dado que el denominador de la última expresión es acotado, mientras que el numerador tiende a 0 cuando $n \rightarrow \infty$, se sigue que el cociente $\left|\tilde{v}_{s}^{n}\right| /\left|\tilde{v}_{u}^{n}\right|$ también tiende a 0 , si $n$ tiende a infinito.

(2): Sean

$$
Q_{n}=\frac{\alpha^{n} \beta^{-n}\left(1+M_{2} \delta_{4}\right) s_{n}}{\left|\left(1-M_{2} \delta_{4}\right)-\beta^{-n} M_{2} \delta_{4} s_{n}\right|}, R_{n}=\frac{\alpha^{n} M_{2} \delta_{4}}{\left|\left(1-M_{2} \delta_{4}\right)-\beta^{-n} M_{2} \delta_{4} s_{n}\right|} .
$$


Puesto que

$$
\frac{\left|\tilde{v}_{s}^{n}\right|}{\left|\tilde{v}_{u}^{n}\right|} \leq Q_{n}+R_{n}
$$

es suficiente ver que cada uno de los sumandos en el miembro derecho de esta última desigualdad converge a 0 , cuando $n$ tiende a infinito. $Q_{n}^{-1}$ :

Es claro que $R_{n} \rightarrow 0$ cuando $n \rightarrow \infty$. En lo que respecta a $Q_{n}$, consideremos primero

$$
\begin{aligned}
Q_{n}^{-1} & =\frac{\left|\left(1-M_{2} \delta_{4}\right)-\beta^{-n} M_{2} \delta_{4} s_{n}\right|}{\alpha^{n} \beta^{-n}\left(1+M_{2} \delta_{4}\right) s_{n}} \\
& \leq \frac{\left(1-M_{2} \delta_{4}\right)}{\alpha^{n} \beta^{-n}\left(1+M_{2} \delta_{4}\right) s_{n}}+\frac{M_{2} \delta_{4}}{\alpha^{n}\left(1+M_{2} \delta_{4}\right) s_{n}}
\end{aligned}
$$

El primer sumando en el miembro derecho de la desigualdad tiende a 0 , mientras que el segundo tiende a $+\infty$, cuando $n \rightarrow \infty$. De esta manera se tiene que $\operatorname{lím}_{n \rightarrow \infty} Q_{n}^{-1}=+\infty$, y en consecuencia $\operatorname{lím}_{n \rightarrow \infty} Q_{n}=0$.

(3): Se prueba en forma similar al caso (1), sólo hace falta observar que en este caso el numerador es acotado, mientras que el denominador tiende a $+\infty$ junto con $n$.

A manera de conclusión debemos hacer un par de observaciones respecto al teorema 7. En primer lugar, el teorema se puede trasladar al semiplano $B_{-}^{u}$, sin necesidad de hacer mayores modificaciones en la demostración. En segundo lugar, hay que mencionar que el teorema admite una generalización "canónica" a dimensiones mayores a 2, una vez hechos los correspondientes cambios en la condición TN de la definición 6 .

\section{Bibliografía}

[Alvarado] W. Alvarado (1983) Gomokliniqeskie kontury avtonomnyh sistem differentsialnyh urabnenii, dissertatsia, Leningradskii Gosudarstvennii Universitet.

[Grobman] D. M. Grobman (1959) O gomeomorfizme sistem differentsialnyh uravnenii, DAN SSSR, 128(5): 880-881.

[Ivanov] B. F. Ivanov (1979) K voprosu o sushestbovanii zamknutyh traektorï v okrestnosti gomokliniqeskoi krivoĭ, Diff. Uravn., 15(8): 1411-1419.

[HARTMAn] P. Hartman (1960) "A lemma in the theory of structural stability of differential equations", Proc. Amer. Math. Soc. 11: 610-620.

[PAlis] J. Palis, "On Morse-Smale dynamical systems", Topology 8(4): 385-404.

[PALIS-De Melo] J. Palis, W. de Melo (1979) Introdução aos sistemas dinâmicos, IMPA, Rio de Janeiro. 